\title{
EDITORIAL
}

\section{Continuous Optimization and Combinatorial Optimization*}

\author{
Liqun $\mathbf{Q I}^{1}$ ， Li-Zhi LIAO ${ }^{2}$, Wenan $\mathrm{ZANG}^{3}$ ， Guanglu ZHOU \\ 1 Department of Applied Mathematics, The Hong Kong Polytechnic University, \\ Hong Kong, China \\ 2 Department of Mathematics, Hong Kong Baptist University, Hong Kong, China \\ 3 Department of Mathematics, University of Hong Kong, Hong Kong, China \\ 4 Department of Mathematics and Statistics, Curtin University of Technology, \\ Perth, WA 6845, Australia
}

(C) Higher Education Press and Springer-Verlag 2009

Optimization is a classical yet fast-growing field. It has been playing vital roles in many areas of Science, Engineering, Business, and our daily life. In recent years, the vast applications of optimization and the tremendous advancement in computing speed (even parallel computers) have created many new research frontiers and challenges both theoretically and numerically for the researchers and practitioners in the field of optimization.

In this special issue, papers in continuous optimization as well as combinatorial optimization cover theoretical investigations, numerical experiments, and practical applications. The paper by Xiaoling $\mathrm{Fu}$ and Bingsheng He studies a class of monotone variational inequalities with linear constraints. Strong theoretical achievement and some efficient numerical scheme have been obtained. Numerical experiments, including some traffic equilibrium problems, are presented. In the paper by Min Li and Xiao-Ming Yuan, they focus on some nonlinear complementarity problems; by using the logarithmic-quadratic proximal method, a new efficient computing scheme is proposed. Solid convergence and attractive numerical results are presented. The paper by Hongyu Zhang and Yiju Wang proposes a new type of solution methods for the split feasibility problem. Its global convergence is proved under mild conditions, and preliminary numerical results are reported to show the efficiency of the proposed method. The next three papers focus on the combinatorial optimization. The paper by Qizhi Fang, Rudolf Fleischer, Jian Li, and Xiaoxun Sun is devoted to core stability

${ }^{*}$ Received September 17, 2009

E-mail: maqilq@polyu.edu.hk, liliao@hkbu.edu.hk,wzang@maths.hku.hk,

G.Zhou@curtin.edu.au 
and some related properties of simple flow games. Structural characterizations of simple networks with these properties are obtained, and efficient algorithms for testing these properties are also devised. The paper by Naoki Katoh, Wencheng Wang, Yinfeng $\mathrm{Xu}$, and Binhai Zhu aims to introduce a useful and powerful tool, the so-called parametric search, in geometric optimization. As illustrations, three original and interesting applications of this method are presented. Finally, in the paper by Weiping Shang, Penjun Wan, and Xiaodong $\mathrm{Hu}$, two approximation algorithms for the minimum broadcast schedule problem in wireless sensor networks are proposed. Some other interesting applications of their novel approach are also discussed.

In summary, we believe that the contributions of the above six papers will offer some insights, recent developments, and applications in the area of the optimization. With more researchers and practitioners joining the community of the optimization, optimization will continue to develop and flourish.

Liqun QI

The Hong Kong Polytechnic University, China

Li-Zhi LIAO

Hong Kong Baptist University, China

Wenan ZANG

University of Hong Kong, China

Guanglu ZHOU

Curtin University of Technology, Australia 